\section{Akutes Lungenversagen: ECMO verringert die 60-Tage-Mortalität}

Munshi L et al. Venovenous extracorporea membrane oxygenation for acute respiratory distress syndrom: a systematic review and metaanalysis. Lancet Respir Med 2019; 7: 163-172

Der Einsatz der venovenösen extrakorporalen Membranoxygenierung (vv-ECMO) bei schwerem akutem Lungenversagen (ARDS) wird kontrovers diskutiert. Bisherige Studien beinhalten zum Teil widersprüchliche Ergebnisse. Kanadisch-US-amerikanische Mediziner brachten in einer systematischen Übersichtsarbeit und Metaanalyse die verfügbare Evidenz auf den aktuellen Stand.

Die Untersuchung berücksichtigte Studien aus den Jahren 2009-2018, die folgende Kriterien erfüllten: Vergleich von mechanischer Ventilation plus vv-ECMO gegenüber alleiniger mechanischer Ventilation mit Versorgung einer refraktären Hypoxie einschließlich inhaliertem Stickstoffmonoxid oder Hochfrequenzoszillation (Kontrollgruppe). Ausgeschlossen waren Studien mit den Schwerpunkten venoarterielle ECMO und extrakorporale Kohlendioxidelimination. Die Auswertung umfasste die beiden randomisierten kontrollierten Studien CESAR (Conventional Ventilator Support vs. Extracorporeal Membrane Oxygenation for Severe Acute Respiratory Failure) und EOLIA (ECMO to Rescue Lung Injury in Severe ARDS) sowie 3 Beobachtungsstudien. Primärer Endpunkt war die 60-Tage-Mortalität aus CESAR und EOLIA. In die Sekundäranalyse gingen die Ergebnisse der randomisierten kontrollierten und der Beobachtungsstudien zum Therapieversagen, zur Mortalität zum Zeitpunkt der letzten Nachbeobachtungsuntersuchung, aus der Mortalitätsanalyse gemäß Behandlung und per Protokoll, die zusammengefasste 30-Tage-Mortalität und unerwünschte Ereignisse ein.

\section{Deutlich geringere Sterb- lichkeit}

Insgesamt konnten L. Munshi et al. aus allen Studien Daten von 1055 Patienten auswerten. 773 Patienten wurden in die Sekundäranalyse eingeschlossen. Die 60Tage-Mortalität betrug in der vv-ECMOGruppe 34\% und in der Kontrollgruppe $47 \%$. Das entsprach einer Risikoreduktion von $27 \%(p=0,008)$. Die Qualität der Evidenz dieses Ergebnisses entsprechend GRADE (Grading of Recommendations Assessment, Development and Evaluation) wurde als moderat beurteilt. In Bezug auf ein Therapieversagen war der Unterschied zugunsten der vv-ECMO noch größer: $36 \%$ vs. $64 \%$, Risikoreduktion $42 \%, p=0,004)$. Sowohl die Analyse gemäß Behandlung als auch per Protokoll zeigten hinsichtlich der Mortalität bei der längsten zur Verfügung stehenden Nachbeobachtung keine signifikanten Unterschiede zwischen der vvECMO- und der Kontrollgruppe. Die 30Tage-Mortalität betrug 31\% (vv-ECMO) und $45 \%$ (Kontrollgruppe) mit einer Risikoreduktion von $31 \%(p=0,02)$. In 19\% der Fälle kam es zu schweren Blutungsereignissen und in $2 \%$ zu kreislaufbedingten oder kanülenassoziierten schweren Komplikationen.

FAZIT

Diese Untersuchung belegt für Patienten mit ARDS einen deutlichen Vorteil hinsichtlich der 60-Tage-Mortalität, wenn sie mit vv-ECMO (und mechanischer Ventilation) anstatt mit alleiniger mechanischer Ventilation behandelt werden. Die vv-ECMO war mit einem moderaten Risiko für schwere Blutungsereignisse verbunden.

Matthias Manych, Berlin 